\title{
Publisher's Note: Nonlocal games and optimal steering at the boundary of the quantum set [Phys. Rev. A 94, 022116 (2016)]
}

\author{
Yi-Zheng Zhen, Koon Tong Goh, Yu-Lin Zheng, Wen-Fei Cao, Xingyao Wu, Kai Chen, and Valerio Scarani \\ (Received 13 September 2016; published 20 September 2016)
}

DOI: 10.1103/PhysRevA.94.039902

This paper was published online on 23 August 2016 with an omission of byline footnotes. Kai Chen's byline footnote should read as "kaichen@ ustc.edu.cn"; Valerio Scarani’s byline footnote should read as "physv@ nus.edu.sg”. The byline footnotes have been added as of 14 September 2016. The byline footnotes are not present in the printed version of the journal. 